\title{
Micro to Nano Integration of Intelligent Wireless Sensors Networks for Structural Meso Scale Applications
}

\author{
A. Amditis \& M. Bimpas ${ }^{1}$, D. Zonta ,M. Pozzi, D. Trapani ${ }^{2}$, D. Ulieru ${ }^{3}, N$. Bertsch $^{4}$, V. Spiering, N. Saillen ${ }^{5}$ \\ J. Santana ${ }^{6}, T$. Sterken, T. Torfs, D. Bairaktaris ${ }^{8}$, S. Camarinopulos, M. Frondistou-Yannas ${ }^{9}$, V.Kalidromitis ${ }^{10^{\prime}}$ \\ Y. Stratakos, ${ }^{1}$ ICCS, National Technical University of Athens, Greece $(s),{ }^{2}$ DIMS, University of Trento, Italy \\ ${ }^{3}$ SITEX 45 SRL, Bucharest, Romania, ${ }^{4}$ MEMSCAP SA, Parc Activillage des Fontaines, ZI Bernin, 38926 \\ Crolles Cedex, France ${ }^{5}$ C2V, P.O. Box 318, 7500 AH Enschede, The Netherlands, ${ }^{6}$ IMEC-NL, Eindhoven, The \\ Netherlands ${ }^{7}$ Imec Belgium, Kapeldreef 75, 3001 Leuven, Belgium, ${ }^{8}$ D. Bairaktaris \& Asc. Ltd, Pesmazoglou \\ $17 A$, Kifissia 14561, Athens, Greece, ${ }^{9}$ RISA Sicherheitsanalysen GmbH, Krumme Straße 55, 10627 Berlin-, \\ Germnany ${ }^{10}$ TECNIC S.p.A., Roma, Italy, ${ }^{11}$ Advanced Microwave Systems Ltd., Athens, Greece \\ ${ }^{1}$ E-mail: dumitru ulieru@yahoo.com.hk
}

\begin{abstract}
Rapid advances in sensing and data transmission techniques, such as Radio Frequency Identification (RFID) technology, Micro-Electro-Mechanical Systems (MEMS), lower power wireless networking and in computation give hopes for a new generation of small, inexpensive, networked sensors that can be distributed on civil and building structures to provide accurate, quantitative information on the physical structure state while in service. This information can be used to assess the structural condition of the monitored facility and aid decision making on rehabilitation so that safety can be attained and rehabilitation costs can be reduced. The aim in EU-funded MEMSCON project was to develop MEMSbased sensors for construction monitoring and to integrate them with a Decision-Support-System (DSS an automatic evaluation software, tol process the measurements and define the condition state, particularly aiming at proactive rehabilitation and rehabilitation after earthquake damage in reinforced concrete buildings. By measuring quantitatively the response of buildings during and after an earthquake, in terms of acceleration and strain become an easy task. based on permanent sensing systems when today the seismic damage is almost exclusively based on visual inspection,.

To overcome these limitations, MEMCON project aims to produce small size sensing nodes, integrating MEMS-based sensors and an RFID tag in a single package that will be attached to reinforced concrete buildings for life-cycle measurements of acceleration and strain; data that will be transmitted to a remote base station using a wireless interface. DSS will accept input from the sensors to assess the structural condition of the monitored building and to select optimal remedial measures.
\end{abstract}

Keywords: Wireless sensors; MEMS RFD`s tag,ASIC, system integration ,Seismic Analysis; Damage detection; Condition state monitoring

\section{Introduction of MEMSCON System}

The MEMSCON Project (www.memscon.com) co-funded by the European Community in the $7^{\text {th }}$ Framework Programme, started in October 2008 till March 2012, involving partners from seven countries The project is devoted to development of a reliable and cost-efficient monitoring system to be integrated in new Reinforced Concrete (RC) buildings for their protection against seismic events and settlement. The system includes a wireless network within the building and a base station linking the building to a remote centre for data interpretation. As outlined in Fig 1, the network includes sensing nodes measuring strain and acceleration measured by dedicated nodes at each level during an earthquake, allowing analysis of the seismic response of the whole structure.
The MEMSCON Project aims to develop a costefficient, effective monitoring system to be installed in new reinforced concrete (RC) buildings, for their protection against earthquake and unforecasted settlement. With this purpose, a new-generation of acceleration and strain sensors based on Micro-ElectroMechanical-Systems (MEMS) and RadioFrequency-Identification (RFID) technologies interfacing with a specifically designed software have been developed within the project.

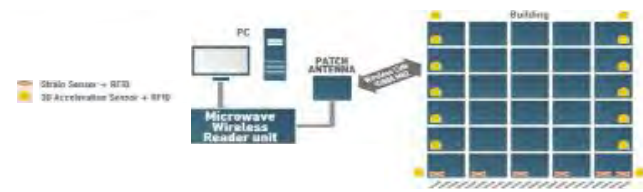

Fig. 1. Scheme of the monitoring system proposed in the MEMSCON project 
The system layout consists of a network of strain and acceleration sensors installed inside the building, recording data when a severe seismic event occurs or when it is scheduled by the user. The sensors transmit data wirelessly to a remote acquisition unit where data are stored, processed and interpreted using the developed software. This software consists of a damage assessment module and a decision support module, providing respectively an estimation of the level of damage inside the building and an insight of the rehabilitation methodologies and costs.

The designed accelerometers are threedimensional capacitive MEMS accelerometers based on different technologies: while for the in plane accelerations ( $X$ and $Y$ channels) the sensor has a interdigitated-comb structure, for the out of plane acceleration ( $Z$ channel) a pendulum system is used The MEMSCON Strain sensors are designed to be directly bonded to the reinforcing bars embedded in the $\mathrm{RC}$ columns at the ground level, namely at the interface between foundation and column. The strain measures, recorded in correspondence of three reinforcing bars inside the element, are used to indirectly estimate the stress inside the whole building, recognizing any stress changes due to unforecasted settlement or overloads. The MEMSCON strain sensor consists of two parts: the front-end sensor embedded into the $\mathrm{RC}$ element and the mote to be attached externally. The front-end sensor is a capacitive MEMS strain sensor connected to an ASIC, both embedded in a PDMS substrate $4 \mathrm{~cm}$ long and $5 \mathrm{~mm}$ thick. The whole package is bonded to a polyimide carrier $8 \mathrm{~cm}$ long and $1 \mathrm{~cm}$ wide, to be bonded in turn to the reinforcing bars, by using a cyanoacrylate glue. The external mote is instead a plastic housing (externally identical to the housing of the accelerometer) inside which there are a lithium battery, a Zigbit module and an antenna. The mote allows for connection up to three strain sensors at a time, in order to reduce to one the required number of devices per columns (Fig.2).

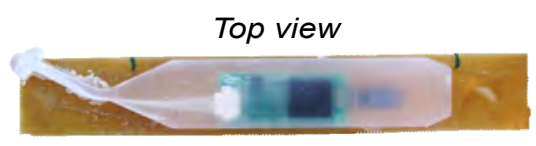

(a)

\section{Bottom view}

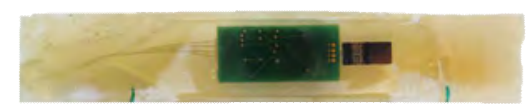

(b)

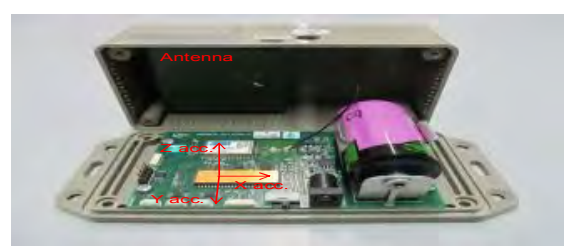

(c)

Fig.1. MEMSCON strain sensor top view (a) and bottom view (b); MEMSCON accelerometer (c)

\section{Strain Sensors Network}

\subsection{Memscon Strain Sensors}

The MEMS strain sensor chip was designed using a capacitive sensing principle. hence a strain in the rebars of the building under study has to be transferred to a change of capacitance in the sensing part of the sensor by a relative displacement of the fingers of a capacitive comb drive structure. the design of the sensor is designed to measure strain over a $+/-30$ '000|je range with a resolution of $10 \mu \mathrm{e}$. it has a size of $3600 \times 8400 \times 1050 \mu \mathrm{m}$.

The MEMS sensor is fabricated using a SOI (Silicon on Insulator) wafer as substrate. SOI wafer consist of a Device layer, a BOX layer and a Handle. Both the Handle and the Device layer are made out of doped Silicon (for the MEMSCON Strain Sensor) and the BOX layer out of Silicon Oxide (glass). The purpose of using such a type of wafer is that it simplifies the production of the moving capacitor (in the Device layer). With such wafers, the desired structure can be patterned in the device layer and released by selectively etching the oxide layer under it.

The layout of the structure is such that 2 differential capacitors are created and each of those capacitors consists of 4 comb drives in parallel. Each comb drives consist of 50 fingers. The dimension of the combidrive is shown hereafter.

The capacitance of a comb drive can be simply calculated from the parallel plate equation. Adapted for the geometry of a comb drive, this equation becomes:

$$
C=n C \cdot 2 \cdot n \cdot \frac{\varepsilon \cdot l \cdot t}{g}=5.31 p F
$$

with e being the permittivity of the medium (eoer)

Two anchors are located on the back side of the MEMS Sensor. They define the position where the strain will be transfer from the rebar to the sensor. The distance between the 2 anchor points define the relation between strain in the structure and displacement in the comb drives. In our case, the distance between the anchors (center to center) is $3 \mathrm{~mm}$, which 
means that the strain of $30^{\prime} 000$ je in the rebar produces a displacement of $90 \mu \mathrm{m}$ in the comb drives.

A challenge was in designing the large stiff spring that holds the moving parts together. an optimum needed to be found between having enough stiffness so that the offset due to packaging and assembly stays within the allowed limit $(20 \mu \mathrm{m})$, not to stiff to limit creep in the glue during operation and finally the stress at full elongation/ compression shall not exceed the yields strength of the silicon.

The theoretical yield strength of monocrystalline silicon is around 7gpa, however, due to defect in the crystal the actual yield strength is closer to 3.5gpa. for the design of the spring, we want to stay in a region of $700 \mathrm{mpa}-1 \mathrm{gpa}$ (53.5 time lower that the actual yield strength) in order to have a sufficient security margin. the design was modeled with a 3D-CAD drawing software (ironcad) and simulations were performed with a finite element model analysis software (Algor) to check that the design doesn't exceed the maximum stress and have enough stiffness. see fig. 4 for the final strain sensor

\section{The accelerometers Sensors Network 3.1 Memscon Accelerometers}

A capacitive sensing principle with mechanical elements realized in a surface micromachining technology has been selected for the MEMSCON accelerometer. The sensor is using independent mechanical elements for each axis. For the in plane sensing, interdigitated comb structures as shown in Fig. 3

The fabrication technology for the three elements is identical; therefore the three elements can be placed on one die as shown in Fig. 4 .

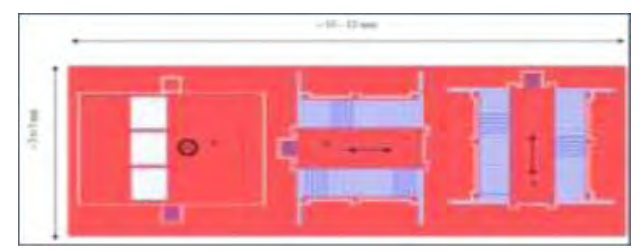

Fig. 3. Concept for the 3D accelerometer

We have also chosen a hybrid integration of the MEMS and its ASIC within a hermetically sealed ceramic package.

For fabrication process the in plane accelerations sensor is using interdigitated comb structures, which form a differential capacitor. The fixed plates of the differential capacitor are formed by fingers attached to the substrate, whereas the movable part is a mass with fingers attached.
Two of these structures rotated by 90 degree's are placed on die.

For the measurement of out of plane accelerations ( $z$ axis) a pendulum with asymmetric mass distribution is used, which is forming the movable part of a differential capacitor (Fig. 4). The fixed part is realized by counter electrode on top of the mechanical element .

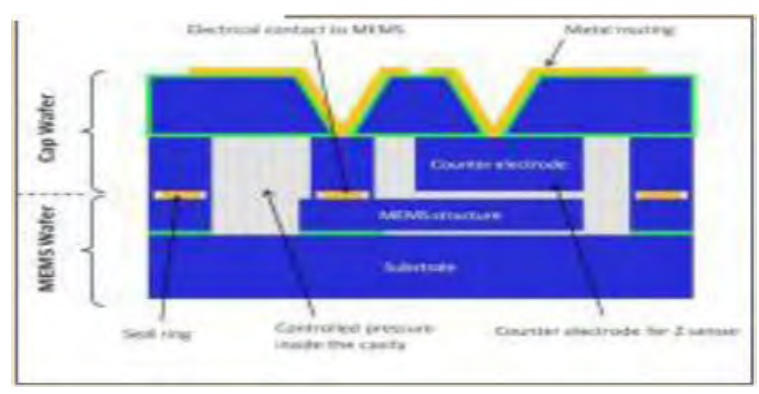

\section{Fig. 4. Schematic sensor cross section}

Although the basic structure of the $x$ - and $y$ sensors and the z-sensor is different, an appropriated design allows the achievement of similar sensor parameters. An SOl wafer is used for the MEMS sensors. The mechanical structures are defined by a combination of DRIE etching and sacrificial layer etching. The cap is formed by two silicon wafers bonded together. The top wafer of the cap stack is etched with $\mathrm{KOH}$ to allow the electrical contact to the cap bottom. For the bottom part of the cap, deep trenches are etched all around the different areas to ensure the electrical isolation. For the top part, the isolation is guaranteed by a thick surface oxidation $(1-2 \mu \mathrm{m})$ of the silicon wafer. The cap itself is mounted to the MEMS wafer by using a metal-silicon eutectic bonding process, used also for electrical connections between the cap and the MEMS wafer.

\section{Memscon System Integration}

The MEMS sensors are integrated into wireless sensor modules as shown in Fig. 5 to form a monitoring system which communicates the measurement data to a remote base station and is controlled by this station:

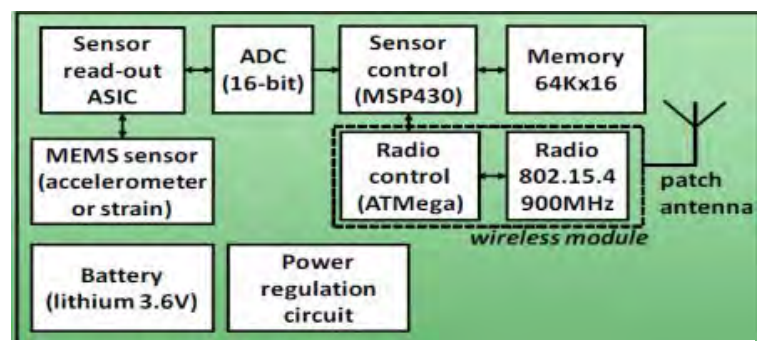

Fig. 5 . Block diagram of the wireless sensor module 
The accelerometers are integrated using standard printed circuit board technology and assembled into a rigid protective housing that allows fixation to the building The wireless communication and processing part is implemented in a similar rigid housing and remains accessible for potential battery replacement. The front-end sensing module combining the MEMS strain sensor, readout ASIC and supporting oscillator circuitry and passives, is embedded inside the concrete structure, applied to the metal reinforcing bars. It uses a special package (fig.6) using PDMS silicone molding and a polyimide carrier in order to allow the sensor to be glued onto the metal bar for efficiently transferring the strain from the metal bar to the MEMS sensor.

For the wireless communication an IEEE 802.15.4 radio module in the $900 \mathrm{MHz}$ band is used in combination with a custom-designed patch antenna, in order to obtain robust links in real-world conditions inside a building. The wireless sensor modules are powered by an 8.5Ah C-cell long operating life primary lithium battery. The modules also include a low power processor and a $64 \mathrm{~K} \times 16$ bit RAM memory to record earthquake events or strain data.

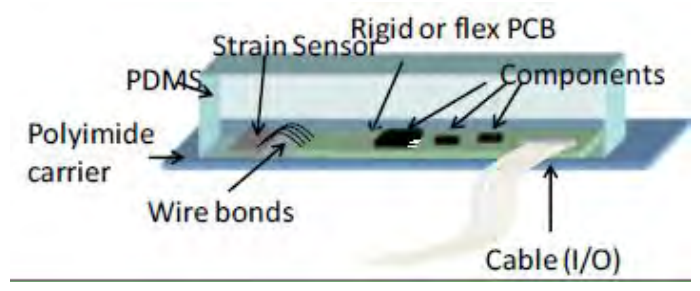

Fig. 6. Strain sensor package

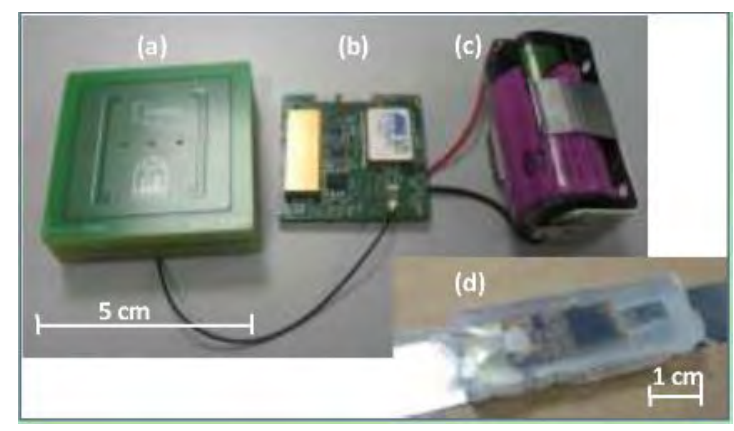

Fig. 7 Picture of the components of the wireless sensor module: (a) antenna, (b) electronics, (c) battery, (d) strain sensor front-end (here on metal rather than polyimide carrier).

A low power network architecture was implemented on top of an 802.15.4 MAC using indirect data transfers which allows the sensor modules' radios to remain powered down most of the time except during brief polling moments and during periodic or even-triggered transmission of recorded data. The strain sensor modules measure periodically and use a radio polling interval of 60 seconds. The $3 D$ accelerometer is constantly running at $3 \mathrm{x}$ $200 \mathrm{~Hz}$ sample rate with the measurements recorded in a 54-second loop buffer required to record the early onset of an earth quake event, even before it reaches a trigger threshold, and requires an ultra low power sensor and readout developed in the MEMSCON project. It also enables the triggering to be done remotely by the base station based on the combined information from several monitoring nodes across the networkeven if locally at some sensor nodes the chosen earthquake threshold was not exceeded. It also allows the wake-up monitoring function to select an amount of sensor modules which do not give many false alarms, e.g. due to street traffic or other local interfering signals., the radio polling interval of the accelerometer modules is limited to 15 seconds.

The strain sensor modules show an average power consumption of $0.274 \mathrm{~mW}$, which results in a operational time of 12 years before the batteries need to be replaced. The accelerometer modules consume $1.73 \mathrm{~mW}$, resulting in 2 years of battery life.

\section{Results Outcomes}

There are two main classes of sensor node hardware: the acceleration sensor nodes and the strain sensor nodes. The strain sensor node can be used in two different applications (measuring strain in the steel or in the concrete), but the hardware and system aspects are identical for both variants.

Some of the systems will function as Zigbee routers, others as Zigbee end-nodes.

\subsection{Acceleration Measurement Scenarios}

The main trigger for the acceleration measurement is the detection of the start of an earthquake. The detection can happen in two ways:

- The output of the built-in accelerometer in a selected number of monitoring nodes exceeds a certain minimum threshold, perhaps during a certain minimum time; these monitoring nodes alert the base station which will decide whether to wake up the entire network of acceleration sensing nodes over the radio

- $\quad$ The monitoring nodes are selected based on their location and amount of environmental noise; ground-level nodes far removed from disturbance sources such as heavy traffic. 
The selection of monitoring nodes is done dynamically from the base station

In both of these cases the wake-up of (most of) the acceleration sensing nodes to initiate measurement has to be done over the radio link. This also wake up the nodes via the base station over the radio link at any chosen time independent of the presence of an earthquake, which is a desired functionality for testability and monitoring of the system as shown on the Figures 8 and 9 and Table $\mathrm{I}$.

The recording of the measurements must start immediately after the wake-up trigger with very low latency (since the earthquake lasts only typ. 15 seconds). The user requirements document specifies a maximum $1 \mathrm{~ms}$ wake-up time. The recording will be 30-60 seconds long (maximum earthquake length) at a sample rate of $200 \mathrm{~Hz}$ according to the user requirements

To achieve the max. $1 \mathrm{~ms}$ latency required, the the accelerometer readout ASIC should have a memory where constantly stores the accelerometer readout data in a first-in-first-out (FIFO) buffer, overwriting old data after a certain time. The maximum wake-up latency of the system must in this case be less than this overwrite time, to allow the woken RFID tag to read out data from the memory buffer in time.

To save power, the accelerometer and readout electronics placed in a low-power loweraccuracy measurement mode prior to the wakeup trigger.

\subsubsection{Accelerometer Sensor Results}

The Acceleration Sensor System has two parts: Measuring part involving the accelerometer, and communicating part involving the transceiver .with battery lifetime: requirements for $>2$ years. The measurement requirements consist of acceleration range: up to $2 \mathrm{~g}$, measurement axis: $x, y, z$ bandwidth: $20 \mathrm{~Hz}$. sampling frequency: $200 \mathrm{~Hz}$ resolution: $0,01 \mathrm{~g}$, accuracy:0.002 g, wake up acceleration level: $20 \mathrm{mg}$, recording time after wake up: $60 \mathrm{sec}$, wake up time: $1 \mathrm{~m}$ sec

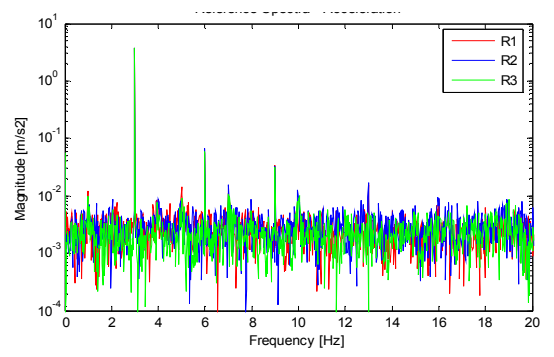

Fig. 8. Example of spectra of references signals

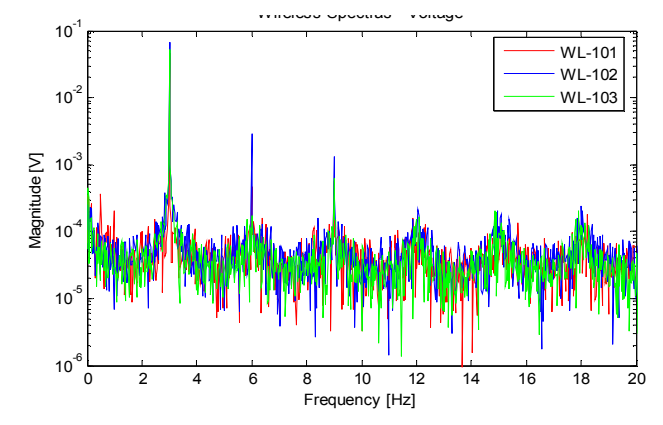

Fig. 9. Example of signals shapes under operation

Table I: Sensitivity curves obtained from calibration tests for the axes of the MEMSCON sensors phase II.1.

\begin{tabular}{|l|l|l|l|}
\hline Features & $\begin{array}{l}\text { Design } \\
\text { specifi } \\
\text { cation }\end{array}$ & $\begin{array}{l}\text { MEMSCON } \\
\text { Phase I }\end{array}$ & $\begin{array}{l}\text { MEMSCON } \\
\text { Phase II.1 }\end{array}$ \\
\hline $\begin{array}{l}\text { Sampling } \\
\text { frequency }\end{array}$ & $200 \mathrm{~Hz}$ & $100 \mathrm{~Hz}$ & $200 \mathrm{~Hz}$ \\
\hline Full scale & $\pm 2 \mathrm{~g}$ & $\pm 1.5 \mathrm{~g}$ & $\pm 3 \mathrm{~g}$ \\
\hline Resolution & $10 \mathrm{mg}$ & $18 \mathrm{mg}$ & $1 \div 4 \mathrm{mg}^{*}$ \\
\hline Noise & - & - & $8 \div 16 \mathrm{mg}$ \\
\hline
\end{tabular}

*depending on the calibration constant.

\subsection{Strain Sensors Measurement Scenarios}

The main measurement scenario for the strain sensor is a periodic readout scenario. A measurement cycle of up to 4 months may be acceptable. This by itself would allow a very low power implementation.

Since manual wake-up functionality from the base station is desired for monitoring and testability reasons, the radio receiver must be enabled as well. Since the wake-up latency can be much higher for this application, a relatively low duty cycle is allowed for the radio receiver.. The results shown on the Figures 10 and 11.

\subsubsection{Strain Sensors Results:}

To assess the performance of the strain monitoring system, three types of tests were performed with the partners of the MEMSCON project:

1.Tensile tests on bare reinforcing bars

2.Tensile tests on reinforced concrete specimens

3.Compression tests on reinforced concrete specimens

The tests confirmed the performance of the monitoring system, testing specimens in laboratory conditions and using materials and construction details in civil reinforced concrete buildings In particular, concrete C25/30 class and B450C steel bars with $20 \mathrm{~mm}$ nominal diameter to assemble the specimens; for three compression In almost all tests, to each reinforcing bar were bonded 2 strain gauges 
connected to 2 wireless strain sensor module and 2 strain gauges connected to a laboratory acquisition device, to a total of four strain gauges on each bar.

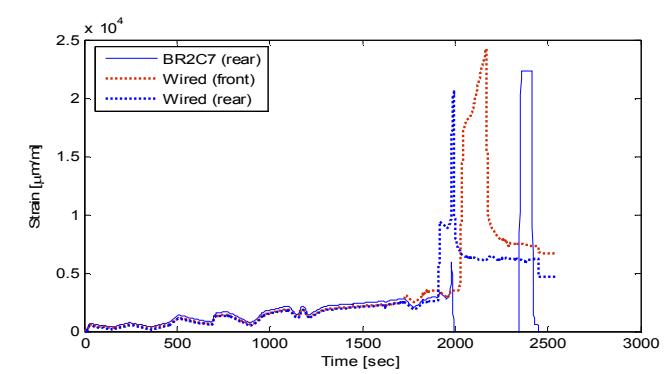

Fig.10.Time histories of strain collected during tensile test

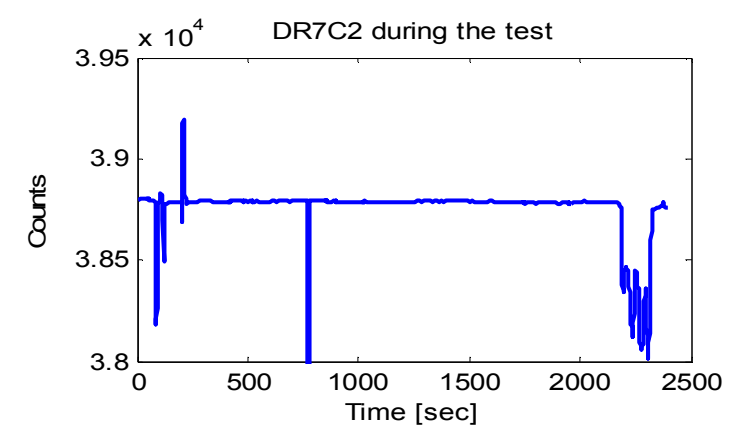

Fig. 11. Diagram of acquisition dates during test

The sensor parameters results are as follows measurement range: $30000 \mu \varepsilon$ (steel), $6000 \mu \varepsilon$ (concrete),accuracy: $10 \mu \varepsilon$ and resolution 10 $\mu \varepsilon$.nominal strength $700 \pm 0.3 \Omega$. Measurement grid thickness $5 \mu \mathrm{m}$ and for ASIC key parameters resulted have a resolution of $1 \mathrm{mg}$ (for accelerometer) and $10 \mu \mathrm{e}(\mathrm{for}$ strain sensor). with $100 \mu \mathrm{A}$ maximum current consumption and linearity $<1 \%$

\section{Conclusions}

Both the strain gauge and the accelerometer networks proved to be reliable under the operational conditions to be expected in a field application. The performance in strain measurement allowed us to follow the onset of yield in the reinforcing steel, which is usually a limitation for sensors applied to civil structures.

The accelerometer network proved to be reliable in the frequency range relevant to seismic analysis, with sufficient accuracy, and several tri-axial nodes can be employed simultaneously. Data acquisition is triggered automatically when a vibration threshold is exceeded, and this allows long-term monitoring, recording all relevant seismic events.
The final products of the MEMSCON project will improve this performance. By development of the sensing nodes reduced the packaging size, the measurement range and reduced power consumption.

The measurements provided by the sensing networks are conceived for post-processing by Decision Supporting System (DSS) which will automatically assess the condition state and suggest an optimal maintenance strategy for the building finalised on the best results and confirmed the major advantages of system concept and high value parameters over competing systems potentially on commercial market.

\section{Acknowledgement}

The authors as partners of MEMSCON project would like to acknowledge the works support of MEMSCON project by European Commission funding on the frame of FP7 Program , Grant Agreement n. CP-TP 212004-

\section{References}

[1] Pozzi M., Zonta D., Trapani D., Amditis A.J., Bimpas M., Stratakos Y.E., Ulieru D., "MEMSbased sensors for post-earthquake damage assessment", Proc. "9th international DAMAS Conference , Oxford, 11-13 July, 2011

[2] Trapani D, Zonta D., Larcher F., Amditis A., Bertsch N., Bimpas M.., Santana J., Sterken T., Stratakos Y., Torfs T., Ulieru D., "Laboratory validation of MEMS-based sensors for postearthquake damage assessment", Lancaster, USA: p. 2165-2172, 2011. Proc. "8th International Workshop on Structural Health Monitoring", Stanford, 13-15 Sep 2011.

[3] Torfs T., Sterken T., Brebels S., Santana J., van den Hoven R., Saillen N, Trapani D., Zonta D., Marmaras P., Bimpas M., Van Hoof C., "Low Power Wireless Sensor Network for Building Monitoring", Proc. IEEE Sensors 2011 Conf., Limerick, 28-31 Oct 2011.

[4] Santana J., Van den Hoven, R., Van Liemp C., Colin M., Saillen N., Trapani D., Zonta D., Torfs T., Van Hoof C. "A 3-axis accelerometer and strain sensor system for building integrity monitoring"Sensors and Actuators A Phys,2011

[5] Colin M., Bertsch N., "3D MEMS accelerometers for building applications. Proc. "1th MEMSCON Workshop" Bucharest, SITEX Edit. $7^{\text {th }}$ Oct 2010.

[6] Santana J., "3-Axis accelerometer and strain sensor readout for MEMS-based capacitive sensors, Proc. "1th MEMSCON Workshop", Bucharest, Edited by SITEX $45,7^{\text {th }}$ Oct 2010. 